\title{
Psoriatic Disease 10 Years Later
}

The concept of psoriatic disease was developed and introduced 10 years $\mathrm{ago}^{1}$. It originated from a better understanding of molecular mechanisms at the basis of the pathogenesis of psoriasis and the associated musculoskeletal manifestations.

In subjects susceptible through a genetic predisposition, in the presence of active environmental factors, an altered immune response induces the inflammation of both skin and musculoskeletal structures, including joints, entheses, synovial sheaths of tendons, as in dactylitis, and the axial skeleton. Tumor necrosis factor- $\alpha$ (TNF- $\alpha$ ) appeared to be the prevalent molecular driver of this inflammation through the activation of epidermal keratinocytes, antigen-presenting dendritic cells, T lymphocytes, endothelial cells, and enthesosynoviocytes. Similar to that of skin and musculoskeletal structures, the microscopic involvement of the bowel described in psoriatic arthritis (PsA) was then included in the concept of psoriatic disease under the same biological propulsion sustained by $\mathrm{TNF}^{2}$. This cytokine, by stimulating the production of growth factors, adhesion molecules, and chemotactic polypeptides, contributes to the recruitment of the cellular network, which colonizes the bowel along with the skin, the joints, and the entheses ${ }^{1}$. Later, in patients with psoriasis, several studies showed an increased prevalence of metabolic syndrome with a consequential increased risk of myocardial infarction and cardiovascular mortality ${ }^{3}$. Thus, over time with the development of new lines of research, psoriatic disease increasingly appeared a systemic condition confined not only to the skin and the musculoskeletal system, but involving several different anatomical areas ${ }^{4,5}$. Along with bowel, the eye, the metabolic profile, and bone metabolism are now fields of growing attention.

Regarding genetic aspects, the dominant psoriatic disease susceptibility sequences have been identified in several regions located within the $\mathrm{MHC}^{6}$. In particular, HLA-Cw0602 has been shown to be associated with cutaneous manifestations and the HLA-B*27 allele with severe articular phenotypes, mainly with the psoriatic spondyloarthritis subset ${ }^{7,8}$.

Other genetic regions have been investigated in the context of multiple and genome-wide association studies. The killer cell Ig-like receptors (KIR), KIR2DS2, expressed on natural killer cells, have been found to be significantly associated to $\mathrm{PsA}^{9}$.

These advancements have improved the understanding on the link between adaptive and innate immunity existing in psoriatic disease, since its first phases.

In fact, Class I HLA molecules, whose involvement is key for antigen presentation, represent a ligand of KIR acting as an adaptive immunity potential driver of innate immunity responses ${ }^{10}$.

Apart from TNF, other cytokines have acquired relevance in the pathogenesis of psoriatic disease. Barnas and Ritchlin have described an interesting mode ${ }^{11}$. They supposed 3 steps of molecular involvement: epidermis, dermis, and lymph node. At the level of epidermis, the model describes the activation of keratinocytes by different stimuli (mechanical and/or infections) with the release of DNA and RNA, which are recognized by plasmocytoid dendritic cells delivering in response to interferon (IFN)- $\alpha$. At the level of dermis, IFN activates dendritic cells, which stimulate draining lymph nodes releasing cytokines influencing $\mathrm{T}$ cell differentiation. Interleukin (IL)-23 triggers the proliferation of IL-17-producing T cells while IL-12 promotes development of IFN-producing T lymphocytes, which migrate back to the skin or colonize the entheses and joints. The consequential release at dermis of IL-17 and IL-22 induces the proliferation of keratinocytes with the production of IL-19 and IL-36 and the release of neutrophil chemotactic factors. The accumulation of neutrophils leads to the formation of Munro's abscesses with the formation of psoriatic plaques ${ }^{11}$.

We have amplified Barnas and Ritchlin's proposal ${ }^{11}$, suggesting a further phase we call the "systemic phase," with the involvement of extracutaneous sites among which we include, along with entheses and joints, the bowel, eye, and adipocytes (Figure 1).

This would better explain the onset of entheso-arthritis, colitis, uveitis, and metabolic syndrome. Our suggestion emphasizes the biological involvement of other cytokines operating with TNF in the development of systemic inflammation, designing a new pathogenetic scenario in which IL-12, IL-17, IL-23, and the axis IL-23/Th17 acquire prominence. Entheso-arthritis is the first extraarticular site of importance. It is the consequence of cytokine involvement of the synovial-entheseal complex, the anatomical entity described by McGonagle, et $a l^{12}$. TNF, IL-17, and the IL-23/Th17 axis play a fundamental role in explaining the different articular features that characterize PsA, including enthesitis, arthritis, and dactylitis ${ }^{13}$. Bone metabolism is affected, too. Receptor activator of nuclear factor- $\kappa \mathrm{B}$ (RANK)/RANK ligand signaling, which regulates activity and differentiation of osteoclasts, has been found altered in PsA synovial tissue. This may occur because the IL-23/17 axis upregulates RANK expression with a possible indirect effect on PsA synovitis ${ }^{14}$.

Personal non-commercial use only. The Journal of Rheumatology Copyright $@$ ( 2017. All rights reserved 


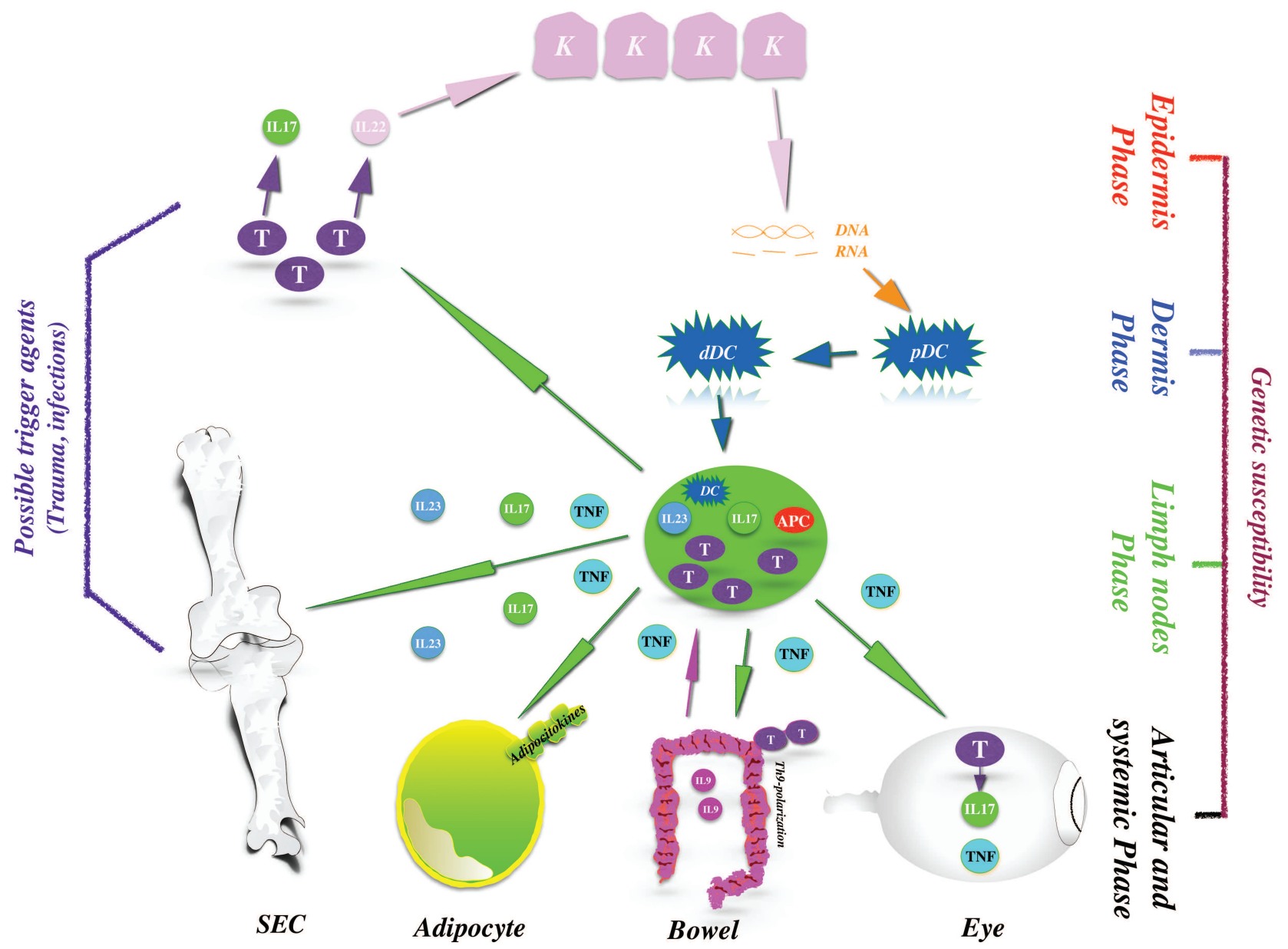

Figure 1. A speculative scheme representing physiopathological mechanisms and clinical manifestations of psoriatic disease. Possible trauma or infective agents trigger at different sites innate and adaptive immune mechanisms in genetically predisposed subjects. In the epidermis phase, T lymphocytes, by IL-17 and IL-22, start the dermis phase with activation of pDC to dDC. Lymph node phase includes APC involvement and activation of T lymphocytes with increased release of proinflammatory cytokines, mainly TNF- $\alpha$, IL-17, and IL-23. These are keys in determining the heterogeneous spectrum of the psoriatic disease, involving eye (uveitis), bowel (colitis), adipocyte (metabolic syndrome), and SEC and the skin itself. A possible link between intestinal and synovial inflammation through IL-9 overexpression and Th9 polarization that occur in synovitis and in the peripheral blood of patients with psoriatic arthritis suggests a potential existence of a bowel-joint migratory axis. Adapted from: Barnas JL, Ritchlin CT. Etiology and pathogenesis of psoriatic arthritis. Rheum Dis Clin North Am 2015;41:643-63. IL: interleukin; pDC: plasmacytoid dendritic cells; dDC: dermal dendritic cells; APC: antigen presenting cells; TNF- $\alpha$ : tumor necrosis factor- $\alpha$; SEC: synovial-entheseal complex; K: keratinocytes.

In the bowel involvement, TNF represents the principal promoter of inflammatory changes. However, several studies have opened new perspectives on the understanding of inflammatory bowel diseases (IBD). In particular, today it is recognized that Th17 cells drive colitis pathogenesis. IL-23 sustains Th17 cell responses and gut inflammation ${ }^{15}$. The introduction of the anti-IL-12/23p40 antibody ustekinumab for the treatment of Crohn disease (CD), approved also for PsA and psoriasis, has contributed to the elucidation of a shared pathway between these pathologies based on the IL-23-driven process ${ }^{16}$.

However, the dual involvement of Th17-related IL-17 cytokines, with protective rather than negative effects in the gut, can be a discriminatory element for differentiating colitis of IBD from PsA colitis ${ }^{15}$. Peculiar balances of intestinal microflora could be involved in the differentiation of these conditions as one of the effects of the different combination of these cytokines produced by Th $17^{17}$. Further, subclinical gut inflammation in patients with PsA has been reported as characterized by a specific histologic and immunologic signature represented by pronounced Paneth cell hyperplasia and Th17 and Th9 responses. Th9 responses have been reported to be a specific PsA signature when compared with ankylosing spondylitis and $\mathrm{CD}^{18}$. A possible link has also been hypothesized between intestinal and synovial inflammation through IL-9 overexpression and Th9 polarization that

$$
\text { Personal non-commercial use only. The Journal of Rheumatology Copyright @ } 2017 \text {. All rights reserved. }
$$


occur in synovitis and in the peripheral blood of patients with PsA. This could suggest a potential existence of a bowel-joint migratory axis ${ }^{18}$.

TNF is a major factor sustaining uveitis ${ }^{19}$, and initial data on the possible involvement of the IL-17 are emerging ${ }^{20}$. In several experimental models, different researchers have shown an increased production of IL-17 during uveitis ${ }^{21,22}$.

The improvement of noninfectious uveitis reported with use of secukinumab could support a role for IL-17A in its pathogenesis in the course of psoriatic disease, but further studies are required ${ }^{23}$.

Finally, we should consider the metabolic syndrome, which develops under the inflammatory propulsion of psoriatic disease. Adipose tissue secretes several adipokines that produce inflammation and metabolic dysfunction ${ }^{24,25,26}$. The rationale sustaining the connection between pathogenetic aspects and systemic involvement is reinforced by studies conducted by the University of Toronto group. In particular, they have highlighted how adipocytokines, metabolic syndrome, and inflammatory severity are correlated in patients with $\mathrm{PsA}^{27}$.

TNF is the most important adipokine, inducing adipocytes apoptosis, insulin resistance, increased plasma levels of triglycerides, and stimulating lipolysis ${ }^{28,29}$. In obese subjects, TNF levels appear increased in the plasma and in adipose tissue $^{27}$. The therapeutic block of TNF has demonstrated the improvement of metabolic syndrome ${ }^{25,26}$, with positive effects on all its different components. The actual scenario is made even more complex by the new components deserving growing attention. The involvement of intracellular enzymes, such as phosphodiesterase 4 (PDE4), and molecules, such as Janus kinase (JAK), playing a key role in cellular signaling, has been characterized in PsA $\mathrm{A}^{30,31,32}$.

The development of therapeutic agents targeting intracellular molecular pathways is a promising opportunity for $\mathrm{PsA}^{33}$. Among them we mention the emerging treatments targeting PDE4 (apremilast) and JAK (tofacitinib) ${ }^{34}$.

The underlying heterogeneity inherent to the systemic expression of psoriatic disease needs to be investigated in depth. This heterogeneity is not fully understood at present, but needs to be considered to optimize the therapeutic approach. Indeed, pathogenetic mechanisms can contribute to differentiated tissue involvements that, in turn, might require a best-targeted approach to the management of the disease ${ }^{35}$.

Because of the heterogeneous molecular background underlying psoriatic disease, the availability of multiple therapeutic approaches would guarantee in the future the possibility of a personalized therapy ${ }^{33}$.

The complexity of the PsA pathogenetic aspects involving multiple cytokines and molecules encourages the continuous collaborative effort of investigation, ranging from molecular research to clinical studies.

After 10 years, the concept of psoriatic disease has gained relevance and may further direct this collaborative effort.
RAFFAELE SCARPA, MD

Associate Professor,

FRANCESCO CASO, $\mathrm{MD}, \mathrm{PhD}$,

Assistant Professor,

LUISA COSTA, MD, PhD,

Assistant Professor,

ROSARIO PELUSO, MD, PhD,

Assistant Professor,

ANTONIO DEL PUENTE, $\mathrm{MD}$,

Assistant Professor,

Rheumatology Unit,

Department of Clinical Medicine and Surgery,

University Federico II,

Naples, Italy;

IGNAZIO OLIVIERI, MD,

Rheumatology Institute of Lucania (IRel), and Rheumatology Department of Lucania, San Carlo Hospital of Potenza

and Madonna delle Grazie Hospital of Matera, and Basilicata Ricerca Biomedica (BRB) Foundation, Potenza, Italy

Address correspondence to Dr. R. Scarpa, Rheumatology Unit, Department of Clinical Medicine and Surgery, University Federico II, Via S. Pansini 5, 80131 Naples, Italy. E-mail: rscarpa@unina.it

\section{REFERENCES}

1. Scarpa R, Ayala F, Caporaso N, Olivieri I. Psoriasis, psoriatic arthritis, or psoriatic disease? J Rheumatol 2006;33:210-2.

2. Scarpa R, Manguso F, D'Arienzo A, D'Armiento FP, Astarita C, Mazzacca G, et al. Microscopic inflammatory changes in colon of patients with both active psoriasis and psoriatic arthritis without bowel symptoms. J Rheumatol 2000;27:1241-6.

3. Mehta NN, Azfar RS, Shin DB, Neimann AL, Troxel AB, Gelfand JM. Patients with severe psoriasis are at increased risk of cardiovascular mortality: cohort study using the General Practice Research Database. Eur Heart J 2010;31:1000-6.

4. Costa L, Caso F, D'Elia L, Atteno M, Peluso R, Del Puente A, et al. Psoriatic arthritis is associated with increased arterial stiffness in the absence of known cardiovascular risk factors: a case control study. Clin Rheumatol 2012;31:711-5.

5. Shang Q, Tam LS, Sanderson JE, Sun JP, Li EK, Yu CM. Increase in ventricular-arterial stiffness in patients with psoriatic arthritis. Rheumatology 2012;51:2215-23.

6. O'Rielly DD, Rahman P. Genetic, epigenetic and pharmacogenetic aspects of psoriasis and psoriatic arthritis. Rheum Dis Clin North Am 2015;41:623-42.

7. Gudjonsson JE, Karason A, Runarsdottir EH, Antonsdottir AA, Hauksson VB, Jónsson HH, et al. Distinct clinical differences between HLA-Cw*0602 positive and negative psoriasis patients - an analysis of 1019 HLA-C- and HLA-B-typed patients. J Invest Dermatol 2006;126:740-5.

8. Haroon M, Winchester R, Giles JT, Heffernan E, FitzGerald O. Certain class I HLA alleles and haplotypes implicated in susceptibility play a role in determining specific features of the psoriatic arthritis phenotype. Ann Rheum Dis 2016;75:155-62.

9. Chandran V, Bull SB, Pellett FJ, Ayearst R, Pollock RA, Gladman DD. Killer-cell immunoglobulin-like receptor gene polymorphisms and susceptibility to psoriatic arthritis. Rheumatology 2014; 53:233-9.

10. Gladman DD. Recent advances in understanding and managing psoriatic arthritis. F1000Res 2016;5:2670.

11. Barnas JL, Ritchlin CT. Etiology and pathogenesis of psoriatic arthritis. Rheum Dis Clin North Am 2015;41:643-63.

Personal non-commercial use only. The Journal of Rheumatology Copyright @ 2017. All rights reserved. 
12. McGonagle D, Aydin SZ, Tan AL. The synovio-entheseal complex and its role in tendon and capsular associated inflammation. J Rheumatol Suppl. 2012 Jul;89:11-4.

13. Durham LE, Kirkham BW, Taams LS. Contribution of the IL-17 pathway to psoriasis and psoriatic arthritis. Curr Rheumatol Rep 2015; 17:55.

14. Ritchlin CT, Haas-Smith SA, Li P, Hicks DG, Schwarz EM. Mechanisms of TNF-alpha- and RANKL-mediated osteoclastogenesis and bone resorption in psoriatic arthritis. J Clin Invest 2003;111:821-31.

15. Sarra M, Pallone F, Macdonald TT, Monteleone G. IL-23/IL-17 axis in IBD. Inflamm Bowel Dis 2010;16:1808-13.

16. Khanna R, Preiss JC, MacDonald JK, Timmer A. Anti-IL-12/23p40 antibodies for induction of remission in Crohn's disease. Cochrane Database Syst Rev 2015;5:CD007572.

17. Puig L. Cytokine pathways and the role of dysbiosis in psoriasis, psoriatic arthritis, and Crohn disease. Actas Dermosifiliogr 2016;107:95-7.

18. Ciccia F, Guggino G, Ferrante A, Raimondo S, Bignone R, Rodolico $\mathrm{V}$, et al. Interleukin-9 overexpression and Th9 polarization characterize the inflamed gut, the synovial tissue, and the peripheral blood of patients with psoriatic arthritis. Arthritis Rheumatol 2016;68:1922-31.

19. Siebert S, Tsoukas A, Robertson J, McInnes I. Cytokines as therapeutic targets in rheumatoid arthritis and other inflammatory diseases. Pharmacol Rev 2015;67:280-309.

20. Oh K, Byoun OJ, Ham DI, Kim YS, Lee DS. Invariant NKT cells regulate experimental autoimmune uveitis through inhibition of Th17 differentiation. Eur J Immunol 2011;41:392-402.

21. Wendling D. Uveitis in seronegative arthritis. Curr Rheumatol Rep 2012;14:402-8.

22. Rosenzweig HL, Galster K, Vance EE, Ensign-Lewis J, Nunez G, Davey MP, et al. NOD2 deficiency results in increased susceptibility to peptidoglycan-induced uveitis in mice. Invest Ophthalmol Vis Sci 2011;52:4106-12.

23. Letko E, Yeh S, Foster CS, Pleyer U, Brigell M, Grosskreutz CL; AIN457A2208 Study Group. Efficacy and safety of intravenous secukinumab in noninfectious uveitis requiring steroid-sparing immunosuppressive therapy. Ophthalmology 2015;122:939-48.

24. Husni ME. Comorbidities in psoriatic arthritis. Rheum Dis Clin North Am 2015;41:677-98.
25. Costa L, Caso F, Ramonda R, Del Puente A, Cantarini L, Darda $\mathrm{MA}$, et al. Metabolic syndrome and its relationship with the achievement of minimal disease activity state in psoriatic arthritis patients: an observational study. Immunol Res 2015;61:147-53.

26. Costa L, Caso F, Atteno M, Del Puente A, Darda MA, Caso P, et al. Impact of 24-month treatment with etanercept, adalimumab, or methotrexate on metabolic syndrome components in a cohort of 210 psoriatic arthritis patients. Clin Rheumatol 2014;33:833-9.

27. Eder L, Jayakar J, Pollock R, Pellett F, Thavaneswaran A, Chandran $\mathrm{V}$, et al. Serum adipokines in patients with psoriatic arthritis and psoriasis alone and their correlation with disease activity. Ann Rheum Dis 2013;72:1956-61.

28. Caso F, Lubrano E, Del Puente A, Caso P, Peluso R, Foglia F, et al. Progress in understanding and utilizing TNF- $\alpha$ inhibition for the treatment of psoriatic arthritis. Expert Rev Clin Immunol 2016;12:315-31.

29. Chimenti MS, Ballanti E, Perricone C, Cipriani P, Giacomelli R, Perricone R. Immunomodulation in psoriatic arthritis: focus on cellular and molecular pathways. Autoimmun Rev 2013;12:599-606.

30. Fiocco U, Martini V, Accordi B, Caso F, Costa L, Oliviero F, et al. Ex vivo signaling protein mapping in T Lymphocytes in the psoriatic arthritis joints. J Rheumatol Suppl. Nov 2015;93:48-52.

31. Fiocco U, Martini V, Accordi B, Caso F, Costa L, Oliviero F, et al. Transcriptional network profile on synovial fluid $\mathrm{T}$ cells in psoriatic arthritis. Clin Rheumatol 2015;34:1571-80.

32. Fiocco U, Accordi B, Martini V, Oliviero F, Facco M, Cabrelle A, et al. JAK/STAT/PKC $\delta$ molecular pathways in synovial fluid T lymphocytes reflect the in vivo $\mathrm{T}$ helper-17 expansion in psoriatic arthritis. Immunol Res 2014;58:61-9.

33. Olivieri I, D'Angelo S, Palazzi C, Padula A. Advances in the management of psoriatic arthritis. Nat Rev Rheumatol 2014; 10:531-42.

34. Caso F, Del Puente A, Peluso R, Caso P, Girolimetto N, Del Puente A, et al. Emerging drugs for psoriatic arthritis. Expert Opin Emerg Drugs 2016;21:69-79.

35. McInnes IB. Psoriatic arthritis: embracing pathogenetic and clinical heterogeneity? Clin Exp Rheumatol 2016;34 Suppl 98:9-11.

J Rheumatol 2017;44:1298-1301; doi:10.3899/jrheum.161402 